
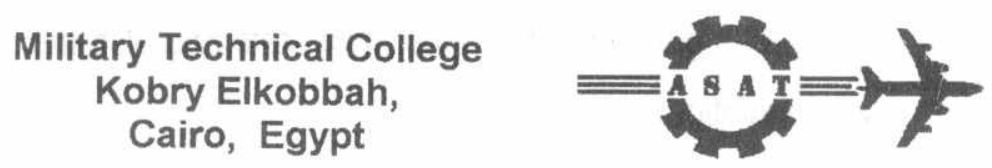
$8^{\text {th }}$ International Conference on Aerospace Sciences \& Aviation Technology

\title{
HYBRID PROPELLANT ROCKET MOTOR
}

\author{
František LUDVÍk*
}

\begin{abstract}
The paper deals with hybrid propellant rocket motor as a propulsion unit of prospective rocket weapon systems, as well as a drive unit of some cosmic rocket carriers.

There are introduced possible constructional schemes of hybrid propellant rocket motor and is mentioned further on the particular general internal ballistic solution of the solid part of the hybrid propellant.
\end{abstract}

The paper shows the advantages and disadvantages of such types of propulsion units.

\section{KEY WORDS}

Hybrid propulsion, hybrid rocket, Internal ballistics, rocket motor.

\section{INTRODUCTION}

The progress in rocketry brings still new tasks, usually complex regarding the requirements laid on rockÆt propulsion units. The mentioned is very important not only from point of view military rocket weapon systems - (RWS), but also from point of view of most sophisticated propulsion systems of cosmic objects.

The hybrid propellant motor - (HPM) regarding the hybrid propellant - (HP) composition is able to fulfill the sever technical and technical requirements demanded for the magnitude of the

* Prof. Dipl.Eng., Ph.D., Department of Rocketry, Military Academy in Brno, Czech Republic. 
specific impulse. Important are also special requirements related to the possibility of HPM restarting during the object flight, especially after longer time of non-operating state and also the more better conditions for the propulsion unit regulation.

Regarding the fact that RWS (and also cosmic objects) still use the classical types of propellants, i.e.

* Solid propellants;

* Liquid propellants.

It can be stated that thrust and mass characteristics of both the propulsion units types are as a rule very near to each others. Therefore it is natural that exist some trials to exploit the propellants having the components in liquid and solid state - HP.

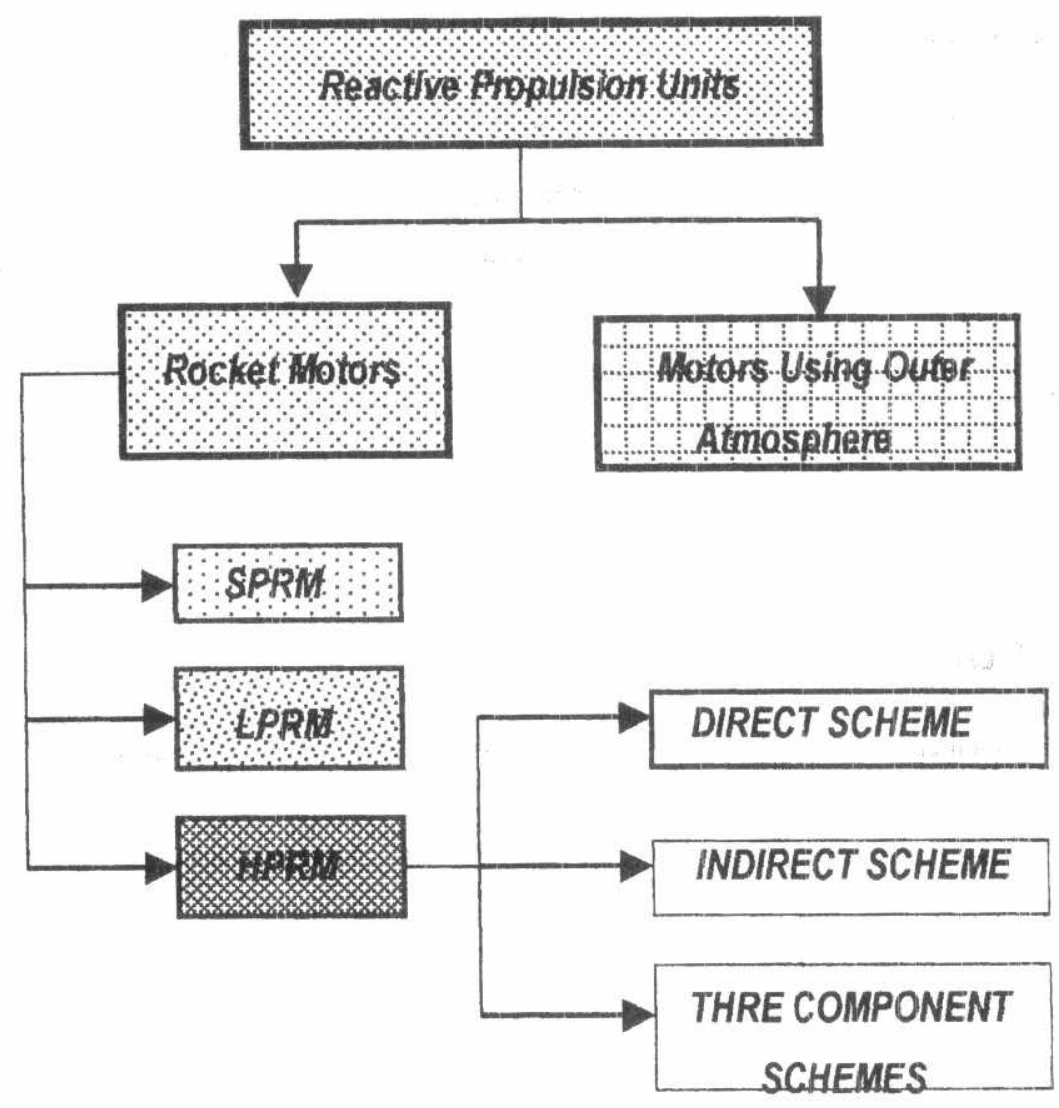

Fig. No. 1. Classification of Reactive Propulsion Units.

General classification of reactive propulsion units can be as follows (see Fig. No. 1.).

HP as mentioned binds together positive features of SP and LP. The following can approve utilization of HP:

* HPM have higher values of specific impulse companing this value with SPRM (consequence of the higher content of chemical energy and higher expansion ratio of combustion products). HPM are simpler in construction when comparing them with LPRM. 
HPM are therefore more reliable in operation, the production price drops and they exploitation is more easy.

* Solid component of the HP practically represents inert agent. Fuel and oxidizer can be produced separately in normal chemical enterprises when using standard production equipment.

* Mechanical properties of HP are as a nule more better when comparing them with SP (due to the smaller content of the fuel binders), meanwhile HP contains higher content of binders, which secures higher mechanical strength, elasticity, etc.

* Buming rate of HP can be regulated by the amount of the second propellant component (liquid). The faults of the solid charge (cracks, cavities etc.) doesn't lead to the burning rate increase, non-controlled working parameters variation. HP have lower sensitivity of the working parameters regarding pressure changes, initial charge temperature variation etc.

* The most effective advantage of HPM is broad scale thrust regulation and possibility of the HPM restarting as well as stopping of its operation during the same mission.

* Working process is more stable. The operational reliability is improved due to the nonpresence of anomalous burning, vibration processes taking place in the HPM combustion chamber.

\section{PRINCIPLE CONSTRUCTIONAL SCHEMES OF HYBRIDPROPELLANT ROCKET MOTORS}

Constructional schemes of HPM are proposed regarding the relation between main working parameters as follows:

* Chamber pressure $p_{C C}=$ cons., mass flow rate of the HP $\dot{m}_{L}=$ cons., mass mixing ratio of HP components $K_{2}=$ cons.

* Chamber pressure $p_{C C}=v a r$., mass flow rate $\dot{m}_{L}=$ cons., mass mixing ratio $K_{2}=\operatorname{var}$.

* Chamber pressure $p_{C C}=v a r$., mass flow rate $\dot{m}_{L}=$ var., mass mixing ratio $K_{2}=$ cons.

The $1^{\text {st }}$ constructional scheme the solid component is a monoblock, having protected nonprofiled fronts and the central channel, which in time $t=0$ is of cylindrical shape. The length of it 
is constant, so that the burning surface is constant value. Due to this all basic working parameters are constant during time of HPM operation (see Fig. No. 2).

HPM designed according to the $2^{\text {nd }}$ constructional scheme has the solid component also in shape of monoblock with cylindrical shape of the channel without non-profiled fronts. Such design secures convenient filling of the combustion chamber - (CC) by the solid component. The mentioned constructional scheme differs from the previous scheme by this property (high filling coefficient - see Fig. No. 3).

HPM designed according to the $3^{\text {rd }}$ constructional scheme has to be proposed so that the mass mixing ratio $K_{2}$ will remain constant there is necessary to vary in certain way the mass flow rate of the liquid component of HP being led inside the central grain channel. Its initial profile has to be profiled (see Fig. No. 4).

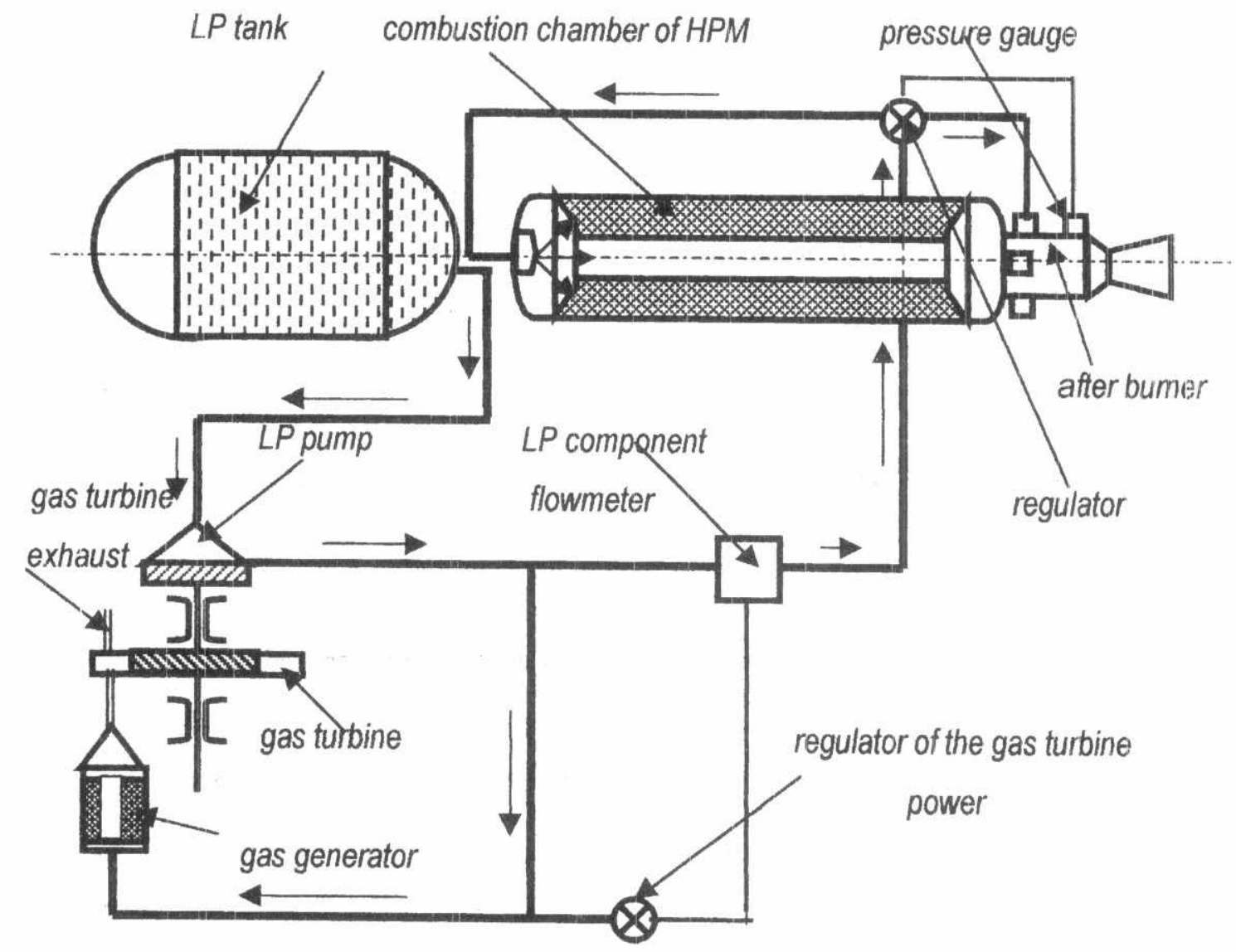

Fig. No. 2. Constructional scheme of HPM - $p_{C C}=$ cons., $\dot{m}_{L}=$ cons. 


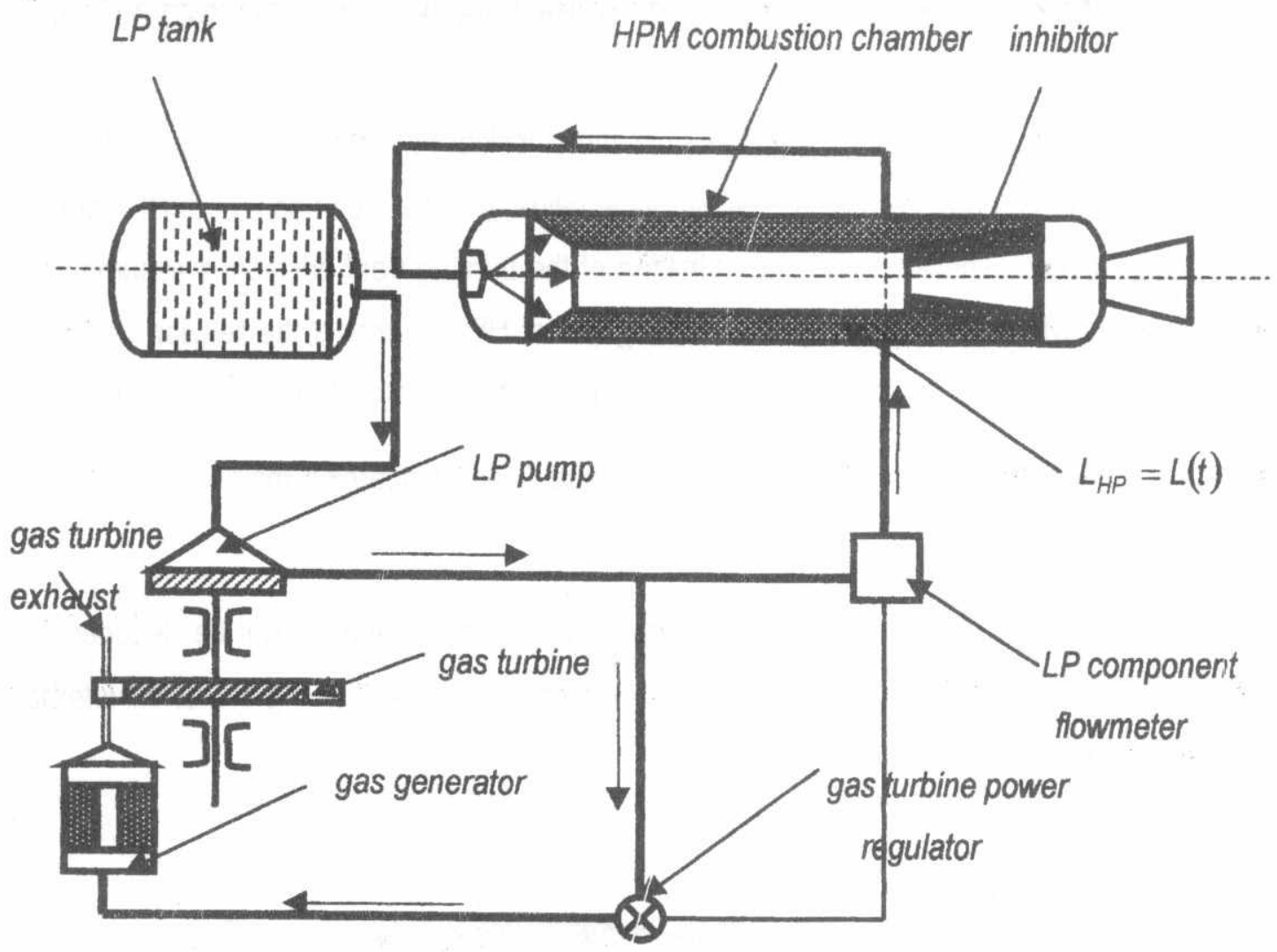

Fig. No. 3. Constructional scheme of HPM $-\dot{m}_{L}=$ cons.

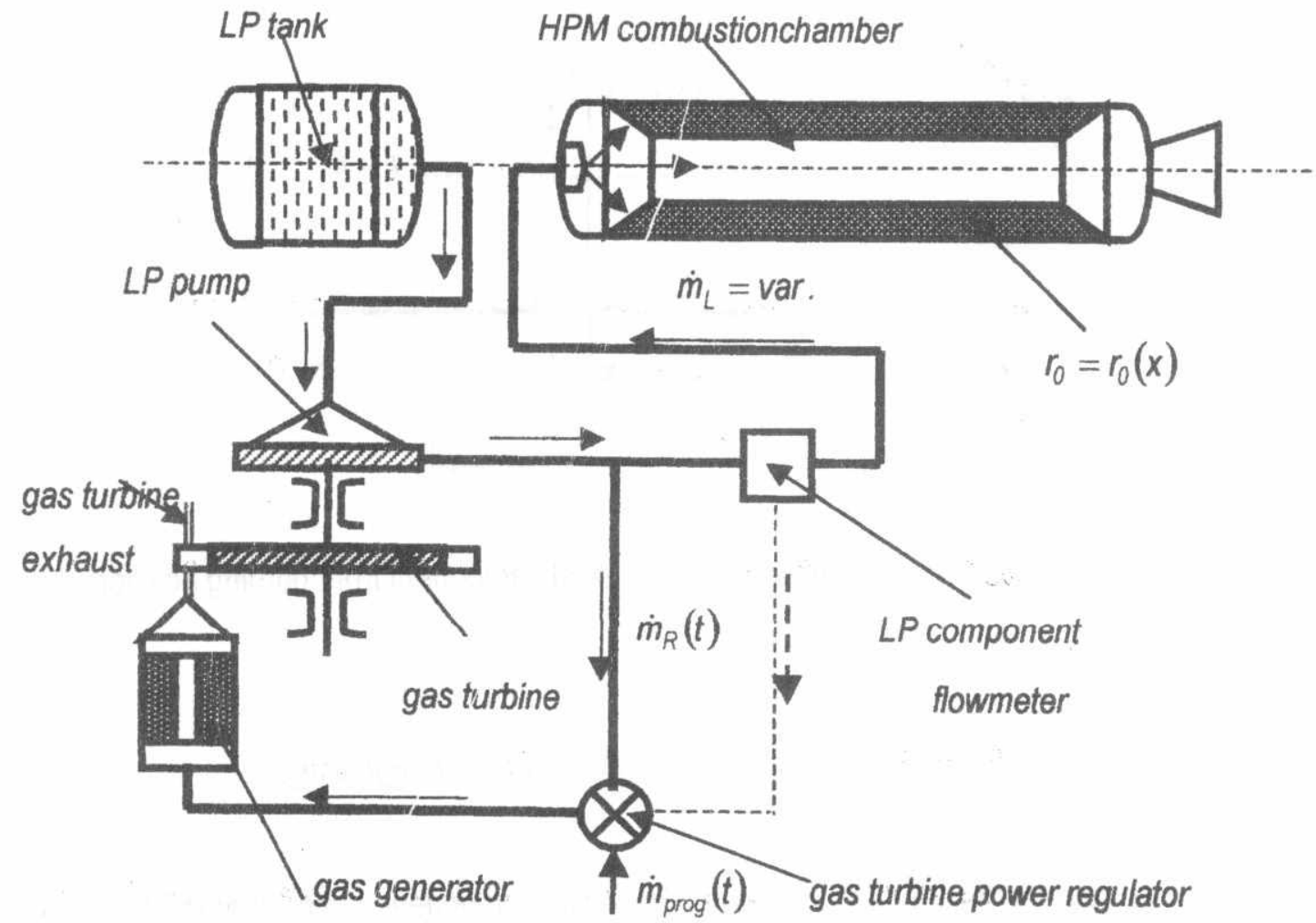

Fig. No. 4. Constructional scheme of $\mathrm{HPM}-\mathrm{K}_{2}=$ cons. 


\section{INTERNAL BALLISTIC SOLUTION OF HYBRID PROPELLANT ROCKET MOTOR}

Internal ballistic solution of HPM differs from the classical RM with e.g. solid propellant in that, that the HP burning rate represents certain "local value" (the solid component of HP doesn't burn in parallel layers). Therefore the determination of the burning front position is assumed as special task of HPM intemal ballistics. The burning law of HP represents the relation between local variations of the internal solid component channel dimensions and local properties of the gas phase with respect to the time, i.e. [1] - $u=\frac{\partial r}{\partial t}$ and $(o w)$ concerning the solid component composition and conditions of its burning.

The equation necessary for the burning front position determination will be deduced for mostly used solid component (grain) shape, i.e. "cylindrical grain shape having cylindrical internal channel" (see Fig. No. 5) [1]

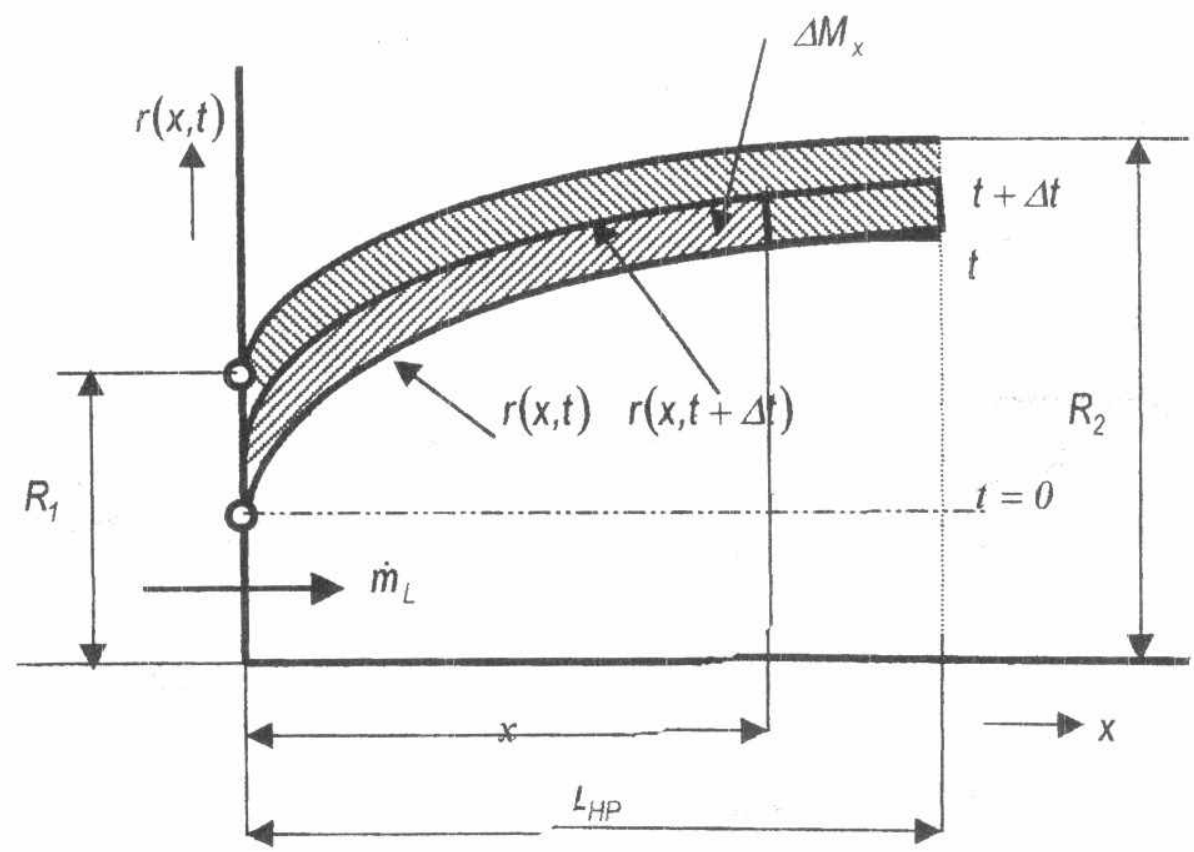

Fig. No. 5. Scharte for determination of the solid component front burning position.

\section{1. DERIVATION OF THE BASIC DIFFERENTIAL EQUATION}

Let $r(x, t)$ represents variable radius of the solid component grain channel, than the equation of the HP burning rate is as follows 


$$
u=\frac{\partial r}{\partial t}=a\left(\frac{\dot{m}_{L}+\dot{m}_{S, x}}{A_{x}}\right)^{v}
$$

where $\underline{a}$ is some constant, $\dot{m}_{L}$ is the mass flow rate of the HP liquid component, $\dot{m}_{S, x}$ is the mass emission of gases from the burning surface of the solid component, $A_{x}$ is the cross-section area of the grain channel in section $\underline{x}$.

The derivative $\frac{\partial r}{\partial t}$ can also be expressed in the following form

$$
\frac{\partial r}{\partial t}=a\left(\frac{\dot{m}_{L}+2 \pi \rho_{S} \int_{0}^{x} r \frac{\partial r}{\partial t} d x}{A_{x}}\right)^{v}
$$

wherefrom also holds

$$
\pi^{v}\left[2 r^{(2 v-1)} \frac{\partial r}{\partial x} \frac{\partial r}{\partial t}+r^{2 v} \frac{\partial^{2} r}{\partial x \partial t}\right]=2 \pi^{v} \rho_{S} a^{\frac{1}{v}} \psi^{(2 v-1)}\left(\frac{\partial r}{\partial t}\right)^{\frac{(2 v-1)}{v}} .
$$

Dividing the equation (3.3) by the term $\pi^{v} r^{(2 v-1)}$ we shall get

$$
r \frac{\partial^{2} r}{\partial x \partial t}+2 v \frac{\partial r}{\partial x} \frac{\partial r}{\partial t}-2 \rho_{S} a^{\frac{1}{v}} v\left(\frac{\partial r}{\partial t}\right)^{\frac{(2 v-1)}{v}}=0
$$

The equation (3.4) is the basic differential equation of the solid component front burning position. The equation holds only for constant or variable value of the mass flow rate of the liquid component of the HP - $\dot{m}_{L}$.

In order to solve the function $r(x, t)$, which determines all HPM main working parameters it is necessary to know the marginal conditions. These conditions allow determining the HPM stable solution. They can be introduced as follows:

1. The values $r$ and $\frac{\partial r}{\partial x}$ for $x=0$ and all values of the time $t(t \geq 0)$;

2. The values $r$ and $\frac{\partial r}{\partial t}$ for $t=0$ and all values of the coordinate $x(x \geq 0)$;

3. The value $r$ for one characteristic of each type.

The marginal condition sub 1. and sub 2. are marginal conditions valid for the solution of so called "Cauchy's task". The marginal condition sub 3. represents "Gauss's condition". 
If the HPM operates at constant value of the liquid component mass flow rate $\dot{m}_{L}=$ cons. and solid component is provided by internal cylindrical channel with initial radius $r_{0}$, than the initial marginal condition is as follows [1]

$$
r(x, 0)=r_{0}=\text { cons. }
$$

The limit marginal condition will be as follows

$$
r(0, t)=r_{1}(t)
$$

The function $r_{1}(t)$ cannot be given arbitrarily, but is ought to be in accordance with the solid component burning law accept. The function $r_{1}(t)$ can be obtained from the equation (3.2) when integrating for $x=0$ and $\dot{m}_{S, x}=0$. The value of $r$ is the function of the time only. The equation (3.2) will than be as follows

$$
\left.\frac{\partial r}{\partial t}\right|_{x=0}=\frac{d r_{1}}{d t}=\frac{\dot{m}_{L}}{\left(\pi r_{1}^{2}\right)^{r}}
$$

After separation of variables and integration we shall get

$$
r_{1}(t)=\left[a\left(\frac{\dot{m}_{L}}{\pi}\right)^{v} t(2 v+1)+r_{0}^{(2 v+1)}\right]^{\frac{1}{(2 v+1)}} .
$$

The marginal conditions given by equation (3.5) and equation (3.7) are marginal conditions of the 1st type.

Let the HPM operates with $\dot{m}_{L}=$ cons. and the solid component of the HP have the above-mentioned shape of the internal grain channel. The outer radius $R$. Initial radius in time $t=$ 0 is $r_{0,1}$. The grain of solid component of the HP has to burn completely if the time of operation will be $t_{k}$. The marginal condition will than be as follows [1]

$$
r\left(x, t_{k}\right)=R=\text { cons. }
$$

The limit marginal condition, i.e. $\underline{r}$ at $\underline{x=0}$ has to be determined from the equation (3.2) as follows

$$
r(0, t)=r_{1}(t)=\left[a\left(\frac{\dot{m}_{L}}{\pi}\right)^{v} t(2 v+1)+r_{0,1}^{(2 v+1)}\right]^{\frac{1}{(2 v+1)}}
$$

The marginal conditions given by equation (3.8) and equation (3.9) are marginal conditions of the $2^{\text {nd }}$ type. 


\section{2. INTERNAL BALL.ISTIC SOLUTION OF THE HYBRID PROPELLANT MOTOR FOR GIVEN}

\section{BURNING LAW EXPONENT}

Analytical solution of this tasks exist only in the case when burning law exponent equals either to $v=0.5$, or $v=1.0$.

General internal ballistic solution as the example will therefore be carried out for $v=0.5$. The equation (3.4) for the introduced burning law exponent (i.e. $v=0.5$ ) can be rewritten as follows

$$
r \frac{\partial^{2} r}{\partial x \partial t}+\frac{\partial r}{\partial x} \frac{\partial r}{\partial t}-a^{2} \rho_{s}=0
$$

Marginal condition given by the equation (3.7) will be then

$$
r(0, t)=\left[2 a t \sqrt{\frac{\dot{m}_{L}}{\pi}}+r_{0}^{2}\right]^{0.5} \text {. }
$$

Marginal condition given by the equation (3.60) will be the same.

Le't us rewrite the equation (3.10) ints the following form, i.e.

$$
\frac{\partial}{\partial t}\left(\frac{\partial r}{\partial x}\right)=a^{2} \rho_{S} \text {. }
$$

The ${ }^{1 s t}$ integral of the equation (3.12) after a simple rearrangement will be

$$
r^{2}=2 a t\left[a \rho_{S} x+\sqrt{\frac{\dot{m}_{L}}{\pi}}\right]+r_{0}^{2} \text {. }
$$

The function $r(x, t)$ will be as follows

$$
r(x, t)=\left[2 a t\left(a \rho_{S} x+\sqrt{\frac{\dot{m}_{L}}{\pi}}\right)+r_{0}^{2}\right]^{0.5} .
$$

The solid component of HP channel will vary during the time of burning. The channel dimensions variation can be determined when differentiating two times the equation (3.12) with respect to variable $x$ and arbitrary time $t$. These derivatives are as follows

$$
\frac{\partial r}{\partial x}=\frac{a^{2} \rho_{S} t}{r} ; \text { and } \frac{\partial^{2} r}{\partial x^{2}}=-\frac{a^{4} \rho_{S} t^{2}}{r^{3}} .
$$

Assuming that $a, \rho_{S}, r$ are positive values in arbitrary time $t$, than holds

$$
\frac{\partial r}{\partial x}>0 \text { and } \frac{\partial^{2} r}{\partial x^{2}}<0
$$


Therefore the solid component channel will vary during its burning (the channel shape enlarges to the end of the solid component grain). The real shape will be convex (see Fig. No. 6 doted line). When such condition will be fulfilled the solid component grain at the end of burning will be completely burnt. The solid component grain profile has to satisfy the following equation

$$
R\left(x, t_{k}\right)=\left[2 a t_{k}\left(a \rho_{S} x+\sqrt{\frac{\dot{m}_{L}}{\pi}}\right)+r_{0}^{2}\right]^{0.5}
$$

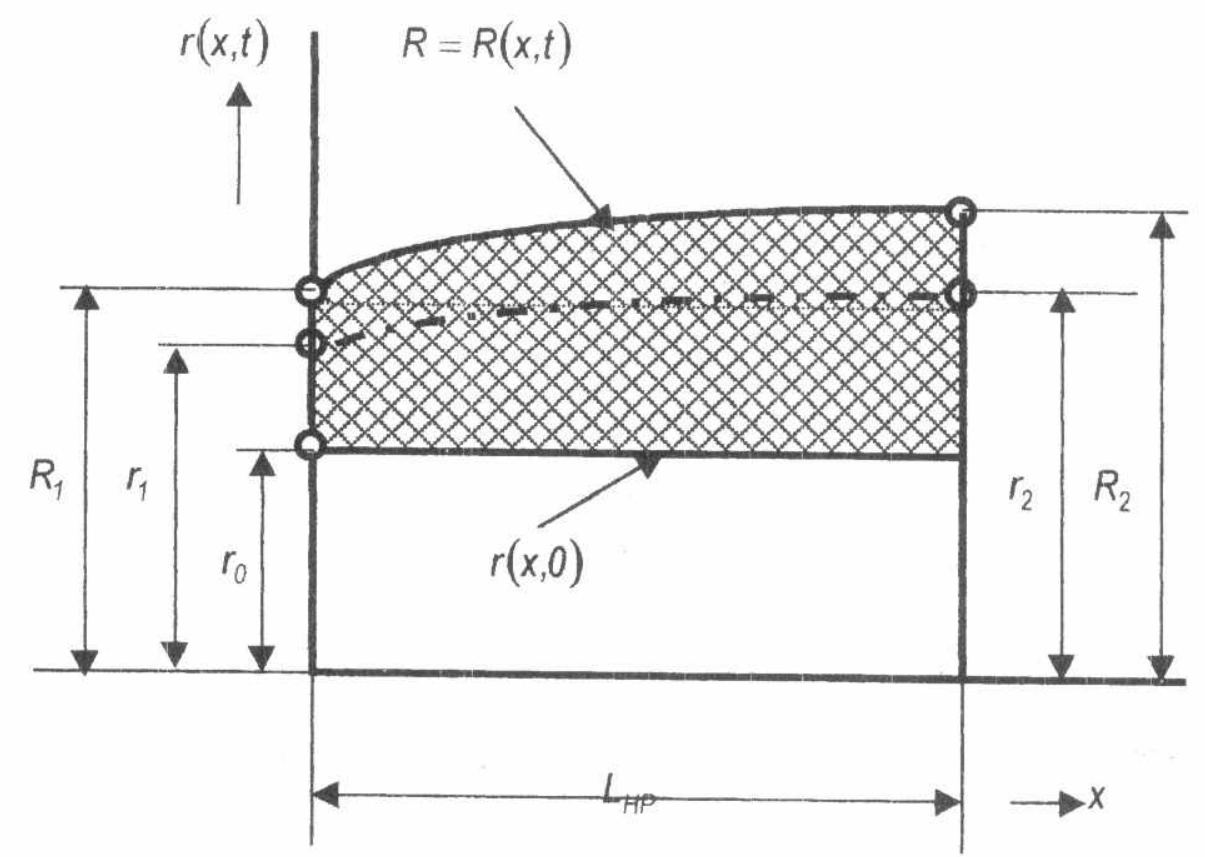

Fig. No. 6. Shape of solid component grain (marginal conditions of the $1^{\text {st type). }}$

Substituting the value $x=0$ and $x=L_{H P}$ the magnitudes of radii at the grain front and aft end will be given as follows

$$
R_{l}=\left(2 a t_{k} \sqrt{\frac{\dot{m}_{L}}{\pi}}+r_{0}^{2}\right)^{0.5}
$$

and

$$
R_{2}=\left[2 a t_{k}\left(a \rho_{S} L_{H P}+\sqrt{\frac{\dot{m}_{L}}{\pi}}\right)+r_{0}^{2}\right]^{0.5}
$$

Mass emission of the solid component of the HP in arbitrary section of the HPM combustion chamber is as follows [1], [3] 


$$
\begin{aligned}
& \dot{m}_{S, x}=2 \pi \rho_{S} \int_{0}^{x}\left(a^{2} \rho_{S} x+a \sqrt{\frac{\dot{m}_{L}}{\pi}}\right) d x=a \rho_{S} x\left(\pi a \rho_{S} x+2 \sqrt{\pi \dot{m}_{L}}\right) \\
& \text { Partial derivative }\left(\frac{\partial r}{\partial t}\right) \text { can be expressed as follows } \\
& \frac{\partial r}{\partial t}=\frac{a\left(a \rho_{S} x+\sqrt{\frac{\dot{m}_{L}}{\pi}}\right)}{r},
\end{aligned}
$$

so that the total mass flow rate of combustion products in arbitrary section of HPM combustion chamber will be

$$
\dot{m}_{\Sigma, x}=\dot{m}_{L}+\dot{m}_{S, x}=\left(\sqrt{\dot{m}_{L}}+\pi^{0.5} a \rho_{S} x\right)^{2}
$$

where $\rho_{S}$ is the density of the solid component of the HP $\left(\mathrm{kgm}^{-3}\right)$.

It is evident from equation (3.18) and equation (3.19) that the magnitudes $\dot{m}_{\sum, x}$ and $\dot{m}_{S, x}$ doesn't depend on time. Therefore if $v=0.5$ and $\dot{m}_{L}=$ cons. will not vary with the time also the mass mixing ratio $K_{x}$.

The value of mass mixing ratio of the HPM designed according to the "direct scheme" (i.e. HPM with liquid oxidizer) will be [1]

$$
K_{x, D}=\frac{\dot{m}_{L}}{\dot{m}_{S, x}}=\frac{1}{\left(1+a \rho_{S} x \sqrt{\frac{\pi}{\dot{m}_{L}}}\right)^{2}-1}
$$

HPM designed according to the "indirect scheme" (i.e. with liquid fuel) the mass mixing ration is as follows

$$
K_{x, I D}=\frac{\dot{m}_{S, x}}{\dot{m}_{L}}=\left(1+a \rho_{S} x \sqrt{\frac{\pi}{\dot{m}_{L}}}\right)^{2}-1
$$

If the mass mixing ratio at the aft end of the solid component of HP is known, i.e. $K_{2}$-given, the length of solid component - $L_{H P}$ is as follows [1]

$$
L_{H P D}=\frac{\sqrt{\frac{K_{2}+1}{K_{2}}}-1}{a \rho_{S} \sqrt{\frac{\pi}{\dot{m}_{L}}}},
$$

or 


$$
L_{H P I D}=\frac{\sqrt{K_{2}+1}-1}{a \rho_{S} \sqrt{\frac{\pi}{\dot{m}_{L}}}} .
$$

If the HPM will have fixed geometry of the nozzle and constant $\dot{m}_{L}$ and $K_{2}$ the chamber pressure $p_{C C}$ (stagnation pressure at the nozzle inlet), specific impulse in vacuum $i_{s V}$ will not vary with respect to the time. Their magnitudes (regardless any losses) are given as follows [1], [3]

$$
\begin{aligned}
& p_{\infty}=\frac{\dot{m}_{\Sigma} c^{*}}{A_{C R}}=\frac{c^{*}\left(\sqrt{\dot{m}_{L}}+\pi^{0.5} a \rho_{S} L_{H P}\right)^{2}}{A_{C R}} ; \\
& i_{s V}=w_{\theta}+\frac{p_{\theta} A_{\theta}}{\dot{m}_{\Sigma, 2}}=w_{e}+\frac{p_{\theta}}{p_{C C}} \frac{A_{\theta}}{A_{C R}} c^{*} ; \\
& F=c_{F} p_{C C} A_{C R},
\end{aligned}
$$

where $p_{\theta}$ is the pressure acting in the exit cross-section of the HPM nozzle, $\frac{p_{\theta}}{p_{C C}}$ is the nozzle expansion ratio, $w_{e}$ is the exit velocity, $c^{*}$ is the characteristic velocity of the used HP and $\left(\frac{A_{\varepsilon}}{A_{C R}}\right)$ is the nozzle enlargement ratio.

If are valid the marginal conditions of the $2^{\text {nd }}$ type there will exist a little different solution.

The marginal condition in this case is as follows

$$
r(0, t)=\left(2 a t \sqrt{\frac{\dot{m}_{L}}{\pi}}+r_{0.1}^{2}\right)^{0.5}
$$

and further on

$$
r\left(0, t_{k}\right)=R=\left(2 a t_{k} \sqrt{\frac{\dot{m}_{L}}{\pi}}+r_{0,1}^{2}\right)^{0.5}
$$

General solution of the differential equation (3.10) with marginal conditions given by equation (3.5) and equation (3.23) will be in the following form

$$
r(x, t)=\left[2 a\left(a \rho_{S}\left\{t-t_{k}\right\} x+t \sqrt{\frac{\dot{m}_{L}}{\pi}}\right)+r_{0,1}^{2}\right]^{0.5} .
$$

In order that the solid component of the HP will burnt fully at the end of the HPM operation the grain channel profile will be as follows 


$$
r(x, 0)=\sqrt{r_{0,1}^{2}-2 a^{2} \rho_{S} x t_{k}} \text {. }
$$

The shape of the channel profile will be of convergent shape (see Fig. No. 7 - convergent in the direction of the $x$ co-ordinate).

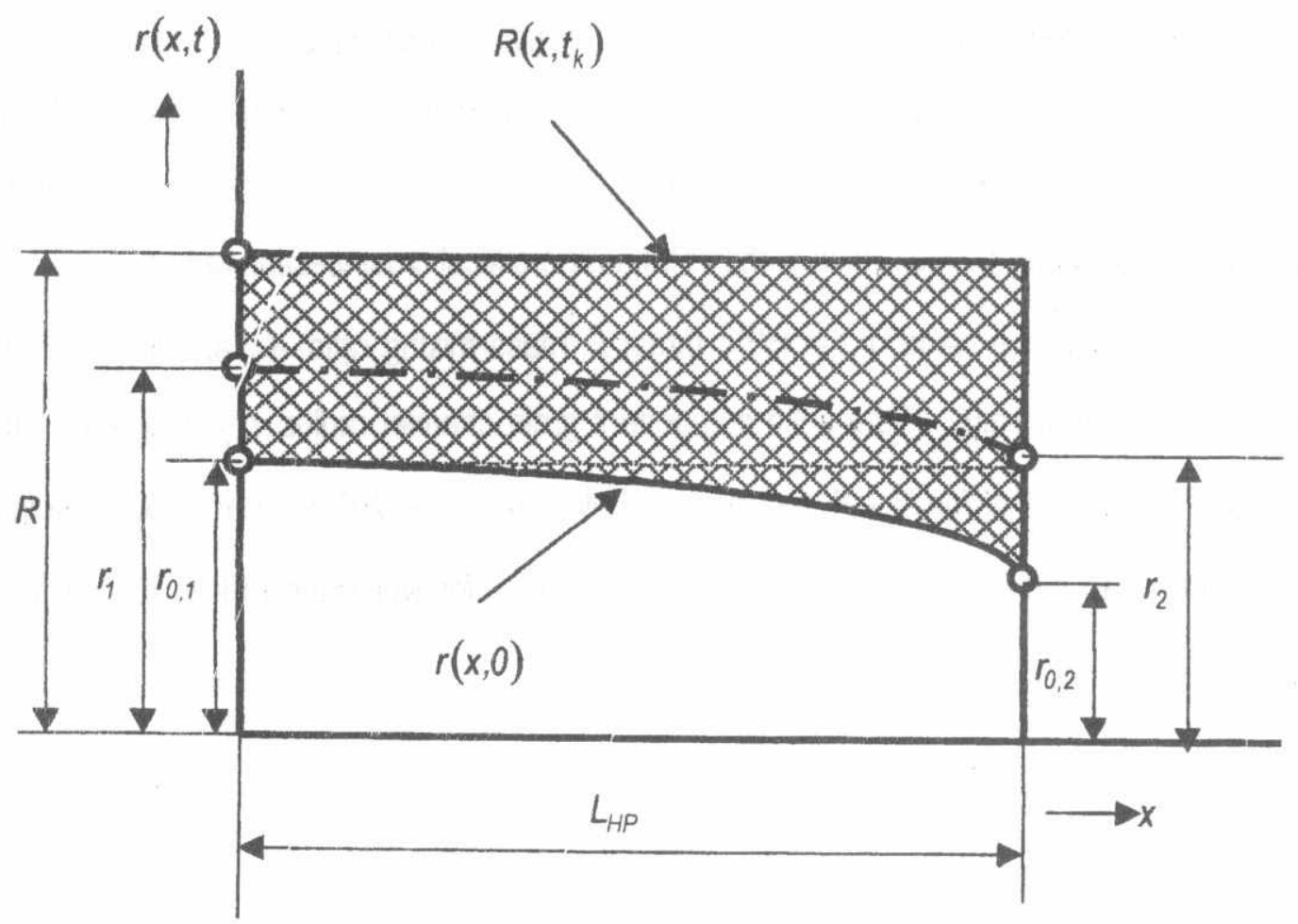

Fig. No. 7. Shape of the HP solid component channel profile (marginal condition of the $2^{\text {nd }}$ type)

For arbitrary $t$ from the interval $0 \leq t \leq t_{k}$ the derivatives $\frac{\partial r}{\partial x}, \frac{\partial^{2} r}{\partial x^{2}}$ are as follows $\frac{\partial r}{\partial x}=\frac{a \rho_{S}\left(t-t_{k}\right)}{r}<0$

ands

$$
\frac{\partial^{2} r}{\partial x^{2}}=-\frac{a^{4} \rho_{S}^{2}\left(t-t_{k}\right)^{2}}{r^{3}}<0
$$

The solid component of the HP due to the above mentioned derivatives will have convex shape (see Fig. No. 7- doted line). The channel shape smoothly decreases regarding the layer burnt and for $t=t_{k}=0$.

Mass emission of the HP solid component in arbitrary section of the HPM combustion chamber in the case discussed will be 


$$
\dot{m}_{S, x}=2 \pi \rho_{S} \int_{0}^{x} r \frac{\partial r}{\partial t} d x=a \rho_{S} x\left(\pi a \rho_{S} x+2 \sqrt{\pi \dot{m}_{L}}\right) .
$$

The result of the equation (3.27) is the same as the result of the equation (3.18), therefore the main parameters of the HPM designed according to the marginal condition of the $2^{\text {nd }}$ type will not vary with respect to the time. The solution of the solid component grain channel profile (cylindrical) van generally is solved as the case when marginal conditions of the $1^{\text {st }}$ type are taken into consideration. It is only necessary to take into consideration the fact, that equation (3.26) doesn't limit the value of the solid component grain radius $-r_{0,2}$. The magnitude of the radius $r_{0,2}$ has not to be greater as the radius of the HPM nozzle critical cross-section radius $r_{C R}$.

Let us assume the value of the HPM thrust $F$, mass mixing ratio $K_{2}$ and time of HPM operation $t_{k}$, than the value of the solid component grain channel radius $r_{0,2}$ is given by the radius of the grain $R$. The functional dependence between $r_{0,2}$ and $R$ is monotonous function. The fact mentioned allows determining the value $R=R_{\text {MIN }}$ for which the radius $r_{0,2}=r_{C R}$. In such case will be valid

$$
R_{\text {MIND }}=\left[r_{\text {CR }}^{2}+2 a t_{k} \sqrt{\frac{\left(K_{2}+1\right) \dot{m}_{L}}{\pi K_{2}}}\right]^{0.5},
$$

or

$$
R_{\text {MINID }}=\left[r_{C R}^{2}+2 a t_{k} \sqrt{\frac{\left(K_{2}+1\right) \dot{m}_{L}}{\pi}}\right]^{0.5} \text {. }
$$

For both the types of HPM constructional arrangement can be used "universal" equation of the minimum radius $R_{\text {MIN }}$, i.e.

$$
R_{M I N}=\left[r_{C R}^{2}+2 a t_{k} \sqrt{\frac{R}{\pi i_{s V}}}\right]^{0.5}
$$

\section{CONCLUSION}

The previous parts of the paper presented introduce the general solution of the internal ballistic solution of the HPM. The result of such solution is usually the necessary solid component grain channel profile (regarding the type of marginal conditions), as well as the HPM working parameters. 
The introduced solution being valid for particular theoretical case (i.e. $v=0.5$ ). A practical value of the HP burning law exponent is usually different from it. The value of the burning law exponent moves with the limits $0.5 \leq v \leq 1.0$.

The general procedure when designing the solid component grain, having cylindrical internal channel can be as follows:

* According to the given thrust and specific impulse and mass mixing ratio of the HP can be determined the mass flow rate of the liquid component of the HP $\dot{m}_{L D}=\frac{K_{2}}{\left(1+K_{2}\right)} \frac{F}{i_{s}} \quad$ (valid for the direct constructional scheme of the HPM) or $\dot{m}_{L D}=\frac{1}{\left(K_{2}+1\right)} \frac{F}{i_{s}}$ (valid for indirect constructional scheme of the HPM).

* Then can be d'atermined the radius $r_{0,1}$ (see equation 3.24). The values $R$ and $t_{k}$ are known values. There has to be fulfilled the condition $R>R_{\text {MIN }}$.

* According to the equation (3.21) or (3.21.a) is determined the needed length of the HP - L LP.

* The iritial solid component grain channel profile is solved according to the equation (3.26), i.e. $r=r(x, 0)$.

* The mass of the solid component of HP is determined according to the following equation - $m_{S}=\pi R^{2} L_{H P} \rho_{S}-\pi \rho_{S} \int_{0}^{L H P} r^{2}(x, 0) d x$ or after some simple rearrangement $m_{S}=\pi \rho_{S} L_{H P}\left(R^{2}-r_{0,1}^{2}\right)+\pi \rho_{S}^{2} a^{2} t_{k} L_{H P}^{2}$.

In a conclusion it can be point out that the introduced solution of the HPM allows to obtain correct and necessary solution, which leads to the real HPM constructional solution.

\section{REFERENCES}

[1] GOLOVKOV,L.G.: Gybridnye raketnye dvigateli (Hybrid Rocket Motors). "VIMO" USSR, Moscow 1976.

[2] GURSA,E. Kurs matematiceskogo analyza (Course of Mathematical Analysis), part III, section I. "GTTI", Moscow 1933.

[3] ALEMASOV,V.E.-DREGALN,A.F.-TISCHIN,A.P.: Teorija raketnych dvigatelej (Theory of Rocket Motors). "Maschinostroenie", Moscow 1969. 